\title{
ON THE NUMBER OF REPRESENTATIONS OF INTEGERS BY CERTAIN QUADRATIC FORMS
}

\author{
SHAUN COOPER
}

(Received 1 October 2007)

\begin{abstract}
Generating functions are used to derive formulas for the number of representations of a positive integer by each of the quadratic forms $x_{1}^{2}+x_{2}^{2}+x_{3}^{2}+2 x_{4}^{2}, x_{1}^{2}+2 x_{2}^{2}+2 x_{3}^{2}+2 x_{4}^{2}, x_{1}^{2}+x_{2}^{2}+2 x_{3}^{2}+4 x_{4}^{2}$ and $x_{1}^{2}+2 x_{2}^{2}+4 x_{3}^{2}+4 x_{4}^{2}$. The formulas show that the number of representations by each form is always positive. Some of the analogous results involving sums of triangular numbers are also given.
\end{abstract}

2000 Mathematics subject classification: primary 11E25; secondary 05A19, 11D85, 33 E05.

Keywords and phrases: sum of squares, sum of triangular numbers, quadratic form, theta function, elliptic function, Lambert series, Lagrange's four squares theorem.

\section{Introduction}

Lagrange's four-squares theorem says that every positive integer may be expressed as a sum of four squares. Jacobi went further and used elliptic functions to prove that the number of solutions of

$$
x_{1}^{2}+x_{2}^{2}+x_{3}^{2}+x_{4}^{2}=n
$$

in integers is

$$
8 \sum_{\substack{d \mid n \\ d \neq 0(\bmod 4)}} d
$$

Since $d=1$ is a divisor of any positive integer $n$, the sum in Jacobi's theorem is positive and Lagrange's theorem follows immediately.

Fine [6, pp. 74-76] proved an analogue of Jacobi's theorem for the quadratic form $x_{1}^{2}+x_{2}^{2}+x_{3}^{2}+2 x_{4}^{2}$, and showed that the number of representations of any positive integer by this form is always positive. The purpose of this paper is to present a short and simple proof of Fine's result, and to obtain analogous results for the quadratic forms $x_{1}^{2}+2 x_{2}^{2}+2 x_{3}^{2}+2 x_{4}^{2}, x_{1}^{2}+x_{2}^{2}+2 x_{3}^{2}+4 x_{4}^{2}$ and $x_{1}^{2}+2 x_{2}^{2}+4 x_{3}^{2}+4 x_{4}^{2}$. The proof depends on simple properties of theta functions and a well-known formula which expresses a difference of two Weierstrass elliptic functions as an infinite product.

(c) 2008 Australian Mathematical Society 0004-9727/08 \$A2.00+0.00 


\section{Notation and lemmas}

Let $q$ be a complex number that satisfies $|q|<1$. Ramanujan's theta functions $\varphi(q)$ and $\psi(q)$ are defined by

$$
\begin{aligned}
& \varphi(q)=\sum_{n=-\infty}^{\infty} q^{n^{2}}, \\
& \psi(q)=\sum_{n=0}^{\infty} q^{n(n+1) / 2} .
\end{aligned}
$$

Some of their basic properties are summarized in the following three lemmas.

LEMMA 2.1.

$$
\begin{aligned}
\varphi(q)+\varphi(-q) & =2 \varphi\left(q^{4}\right), \\
\varphi(q)-\varphi(-q) & =4 q \psi\left(q^{8}\right), \\
\varphi(q)^{2}+\varphi(-q)^{2} & =2 \varphi\left(q^{2}\right)^{2}, \\
\varphi(q)^{2}-\varphi(-q)^{2} & =8 q \psi\left(q^{4}\right)^{2} .
\end{aligned}
$$

PROOF. These can be proved using series manipulations. A complete proof is given in [3, pp. 40-41].

LEMMA 2.2.

$$
\begin{aligned}
\varphi(-q) & =\prod_{j=1}^{\infty} \frac{\left(1-q^{j}\right)^{2}}{\left(1-q^{2 j}\right)} \\
\psi(q) & =\prod_{j=1}^{\infty} \frac{\left(1-q^{2 j}\right)^{2}}{\left(1-q^{j}\right)} .
\end{aligned}
$$

ProOF. These are consequences of the Jacobi triple product identity. See [3, pp. 36-37] for proofs.

LEMMA 2.3.

$$
\begin{aligned}
\varphi\left(-q^{2}\right)^{2} & =\varphi(q) \varphi(-q), \\
\psi(q)^{2} & =\varphi(q) \psi\left(q^{2}\right) .
\end{aligned}
$$

PROOF. These are consequences of Lemma 2.2. See [3, p. 40] for proofs.

The final lemma in this section, and the main tool for this work, is a classical result that expresses a quotient of Weierstrass sigma functions as a difference of two Weierstrass elliptic functions. 
LEMMA 2.4. Let $x, z$ and $q$ be any complex numbers satisfying $|q|<|x|,|z|<|q|^{-1}$, and $x, z \neq 1$. Then

$$
\begin{aligned}
& \frac{(1-x z)(x-z)}{(1-x)^{2}(1-z)^{2}} \\
& \times \prod_{j=1}^{\infty} \frac{\left(1-x z q^{j}\right)\left(1-q^{j} x / z\right)\left(1-q^{j} z / x\right)\left(1-q^{j} /(x z)\right)\left(1-q^{j}\right)^{4}}{\left(1-x q^{j}\right)^{2}\left(1-z q^{j}\right)^{2}\left(1-q^{j} / x\right)^{2}\left(1-q^{j} / z\right)^{2}} \\
& \quad=\frac{x}{(1-x)^{2}}-\frac{z}{(1-z)^{2}}+\sum_{j=1}^{\infty} \frac{j q^{j}}{1-q^{j}}\left(x^{j}+x^{-j}-z^{j}-z^{-j}\right) .
\end{aligned}
$$

PROOF. There are many proofs of this result in the literature. For a simple one, see [5]. For an equivalent statement in terms of the Weierstrass functions, see [7, p. 451, Exercise 1]. For generalizations, see [4].

We shall use the following special values of Jacobi's symbol:

$$
\left(\frac{2}{n}\right)=\left\{\begin{aligned}
1 & \text { if } n \equiv 1 \text { or } 7(\bmod 8) \\
-1 & \text { if } n \equiv 3 \text { or } 5(\bmod 8) \\
0 & \text { otherwise }
\end{aligned}\right.
$$

where $n$ is a positive integer. The Jacobi symbol is multiplicative, that is,

$$
\left(\frac{2}{m n}\right)=\left(\frac{2}{m}\right)\left(\frac{2}{n}\right)
$$

for all positive integers $m$ and $n$.

\section{Theta function identities}

The results in this section all hinge on the following result.

THEOREM 3.1.

$$
\begin{aligned}
\varphi(-q) \varphi\left(-q^{2}\right) \varphi\left(-q^{4}\right)^{2} & =1-2 \sum_{j=1}^{\infty}\left(\frac{2}{j}\right) \frac{j q^{j}}{1-q^{j}} \\
q \psi(q)^{2} \psi\left(q^{2}\right) \psi\left(q^{4}\right) & =\sum_{j=1}^{\infty} \frac{j\left(q^{j}-q^{3 j}-q^{5 j}+q^{7 j}\right)}{1-q^{8 j}}
\end{aligned}
$$

PROOF. Let $\omega=\exp (i \pi / 4)$ and take $x=\omega^{3}, z=\omega$, in Lemma 2.4 to get

$$
\begin{aligned}
& \frac{2\left(\omega^{3}-\omega\right)}{(1-\omega)^{2}\left(1-\omega^{3}\right)^{2}} \prod_{j=1}^{\infty} \frac{\left(1+q^{j}\right)^{2}\left(1+q^{2 j}\right)\left(1-q^{j}\right)^{4}}{\left(1-\omega q^{j}\right)^{2}\left(1-\omega^{3} q^{j}\right)^{2}\left(1-\omega^{5} q^{j}\right)^{2}\left(1-\omega^{7} q^{j}\right)^{2}} \\
& =\frac{\omega^{3}}{\left(1-\omega^{3}\right)^{2}}-\frac{\omega}{(1-\omega)^{2}}+\sum_{j=1}^{\infty} \frac{j q^{j}}{1-q^{j}}\left(\omega^{3 j}+\omega^{-3 j}-\omega^{j}-\omega^{-j}\right) .
\end{aligned}
$$


By straightforward calculations,

$$
\frac{2\left(\omega^{3}-\omega\right)}{(1-\omega)^{2}\left(1-\omega^{3}\right)^{2}}=\frac{\omega^{3}}{\left(1-\omega^{3}\right)^{2}}-\frac{\omega}{(1-\omega)^{2}}=\sqrt{2}
$$

Next, using the algebraic rearrangements

$$
1+x=\frac{1-x^{2}}{1-x}
$$

and

$$
(1-\omega x)\left(1-\omega^{3} x\right)\left(1-\omega^{5} x\right)\left(1-\omega^{7} x\right)=\frac{1-x^{8}}{1-x^{4}},
$$

the left-hand side of (3.1) simplifies to

$$
\sqrt{2} \prod_{j=1}^{\infty} \frac{\left(1-q^{j}\right)^{2}\left(1-q^{2 j}\right)\left(1-q^{4 j}\right)^{3}}{\left(1-q^{8 j}\right)^{2}}
$$

which, by (2.5), equals

$$
\sqrt{2} \varphi(-q) \varphi\left(-q^{2}\right) \varphi\left(-q^{4}\right)^{2} .
$$

Furthermore, by checking the residue classes modulo 8 , we find that

$$
\omega^{3 j}+\omega^{-3 j}-\omega^{j}-\omega^{-j}=-2 \sqrt{2}\left(\frac{2}{j}\right) .
$$

Therefore, the right-hand side of (3.1) simplifies to

$$
\sqrt{2}-2 \sqrt{2} \sum_{j=1}^{\infty}\left(\frac{2}{j}\right) \frac{j q^{j}}{1-q^{j}} .
$$

Combining (3.2) and (3.3), we complete the proof of the first result.

Now let us prove the second result. In Lemma 2.4 , replace $(q, x, z)$ with $\left(q^{8}, q, q^{3}\right)$ to get

$$
\begin{aligned}
q & \prod_{j=1}^{\infty} \frac{\left(1-q^{8 j-4}\right)^{2}\left(1-q^{8 j-6}\right)\left(1-q^{8 j-2}\right)\left(1-q^{8 j}\right)^{4}}{\left(1-q^{8 j-7}\right)^{2}\left(1-q^{8 j-5}\right)^{2}\left(1-q^{8 j-3}\right)^{2}\left(1-q^{8 j-1}\right)^{2}} \\
& =\frac{q}{(1-q)^{2}}-\frac{q^{3}}{1-q^{3}}+\sum_{j=1}^{\infty} \frac{j q^{8 j}}{1-q^{8 j}}\left(q^{j}+q^{-j}-q^{3 j}-q^{-3 j}\right)
\end{aligned}
$$

The left-hand side of (3.4) simplifies to

$$
q \prod_{j=1}^{\infty} \frac{\left(1-q^{2 j}\right)^{3}\left(1-q^{4 j}\right)\left(1-q^{8 j}\right)^{2}}{\left(1-q^{j}\right)^{2}}
$$


which, by (2.6), equals

$$
q \psi(q)^{2} \psi\left(q^{2}\right) \psi\left(q^{4}\right)
$$

The right-hand side of (3.4) is

$$
\begin{aligned}
& \sum_{j=1}^{\infty} j\left(q^{j}-q^{3 j}\right)+\sum_{j=1}^{\infty} \sum_{k=1}^{\infty} j\left\{q^{(8 k+1) j}+q^{(8 k-1) j}-q^{(8 k+3) j}-q^{(8 k-3) j}\right\} \\
& \quad=\sum_{j=1}^{\infty} \sum_{k=1}^{\infty} j\left\{q^{(8 k-7) j}+q^{(8 k-1) j}-q^{(8 k-5) j}-q^{(8 k-3) j}\right\} \\
& \quad=\sum_{j=1}^{\infty} \frac{j\left(q^{j}-q^{3 j}-q^{5 j}+q^{7 j}\right)}{1-q^{8 j}} .
\end{aligned}
$$

Combining (3.5) and (3.6), we complete the proof of the second result.

The next theorem gives Lambert series expansions for four different products of theta functions.

THEOREM 3.2. Let

$$
f_{1}(q)=1-2 \sum_{j=1}^{\infty}\left(\frac{2}{j}\right) \frac{j q^{j}}{1-q^{j}} \quad \text { and } \quad f_{2}(q)=\sum_{j=1}^{\infty} \frac{j\left(q^{j}-q^{3 j}-q^{5 j}+q^{7 j}\right)}{1-q^{8 j}}
$$

Then

$$
\begin{aligned}
\varphi(q)^{3} \varphi\left(q^{2}\right) & =f_{1}(q)+8 f_{2}(q), \\
\varphi(q) \varphi\left(q^{2}\right)^{3} & =f_{1}(q)+4 f_{2}(q), \\
\varphi(q)^{2} \varphi\left(q^{2}\right) \varphi\left(q^{4}\right) & =f_{1}\left(q^{2}\right)+4 f_{2}(q), \\
\varphi(q) \varphi\left(q^{2}\right) \varphi\left(q^{4}\right)^{2} & =f_{1}\left(q^{2}\right)+2 f_{2}(q) .
\end{aligned}
$$

PROOF. By (2.4) followed by two applications of each of (2.7) and (2.8),

$$
\begin{aligned}
\varphi(q)^{3} \varphi\left(q^{2}\right) & =\varphi(q) \varphi\left(q^{2}\right)\left\{\varphi(-q)^{2}+8 q \psi\left(q^{4}\right)^{2}\right\} \\
& =\varphi(-q) \varphi\left(-q^{2}\right)^{2} \varphi\left(q^{2}\right)+8 q \varphi(q) \psi\left(q^{2}\right)^{2} \psi\left(q^{4}\right) \\
& =\varphi(-q) \varphi\left(-q^{2}\right) \varphi\left(-q^{4}\right)^{2}+8 q \psi(q)^{2} \psi\left(q^{2}\right) \psi\left(q^{4}\right) .
\end{aligned}
$$

Similarly, by (2.3) and (2.4), followed by two applications of each of (2.7) and (2.8),

$$
\begin{aligned}
\varphi(q) \varphi\left(q^{2}\right)^{3} & =\varphi(q) \varphi\left(q^{2}\right)\left\{\varphi(-q)^{2}+4 q \psi\left(q^{4}\right)^{2}\right\} \\
& =\varphi(-q) \varphi\left(-q^{2}\right)^{2} \varphi\left(q^{2}\right)+4 q \varphi(q) \psi\left(q^{2}\right)^{2} \psi\left(q^{4}\right) \\
& =\varphi(-q) \varphi\left(-q^{2}\right) \varphi\left(-q^{4}\right)^{2}+4 q \psi(q)^{2} \psi\left(q^{2}\right) \psi\left(q^{4}\right) .
\end{aligned}
$$


By (2.2) followed by three applications of each of (2.7) and (2.8),

$$
\begin{aligned}
\varphi(q)^{2} \varphi\left(q^{2}\right) \varphi\left(q^{4}\right) & =\varphi(q) \varphi\left(q^{2}\right) \varphi\left(q^{4}\right)\left\{\varphi(-q)+4 q \psi\left(q^{8}\right)\right\} \\
& =\varphi\left(-q^{2}\right)^{2} \varphi\left(q^{2}\right) \varphi\left(q^{4}\right)+4 q \varphi(q) \varphi\left(q^{2}\right) \psi\left(q^{4}\right)^{2} \\
& =\varphi\left(-q^{2}\right) \varphi\left(-q^{4}\right)^{2} \varphi\left(q^{4}\right)+4 q \varphi(q) \psi\left(q^{2}\right)^{2} \psi\left(q^{4}\right) \\
& =\varphi\left(-q^{2}\right) \varphi\left(-q^{4}\right) \varphi\left(-q^{8}\right)^{2}+4 q \psi(q)^{2} \psi\left(q^{2}\right) \psi\left(q^{4}\right) .
\end{aligned}
$$

Similarly, by (2.1) and (2.2), followed by three applications of each of (2.7) and (2.8), we have (omitting some details which are similar to the above)

$$
\begin{aligned}
\varphi(q) \varphi\left(q^{2}\right) \varphi\left(q^{4}\right)^{2} & =\varphi(q) \varphi\left(q^{2}\right) \varphi\left(q^{4}\right)\left\{\varphi(-q)+2 q \psi\left(q^{8}\right)\right\} \\
& =\varphi\left(-q^{2}\right) \varphi\left(-q^{4}\right) \varphi\left(-q^{8}\right)^{2}+2 q \psi(q)^{2} \psi\left(q^{2}\right) \psi\left(q^{4}\right)
\end{aligned}
$$

Substituting the results of Theorem 3.1 into the right-hand sides of (3.7)-(3.10), we complete the proof.

Results involving the function $\psi(q)$ (and sometimes $\varphi(q)$ as well) can be obtained by dissecting one of the results in Theorem 3.1. These are given in the following result.

THEOREM 3.3.

$$
\begin{aligned}
q \psi\left(q^{8}\right) \varphi\left(q^{8}\right)^{3} & =\sum_{n=0}^{\infty}\left(\sum_{d \mid 8 n+1}\left(\frac{2}{d}\right) d\right) q^{8 n+1} \\
q^{3} \psi\left(q^{8}\right)^{3} \varphi\left(q^{8}\right) & =-\frac{1}{2} \sum_{n=0}^{\infty}\left(\sum_{d \mid 8 n+3}\left(\frac{2}{d}\right) d\right) q^{8 n+3} \\
q^{5} \psi\left(q^{8}\right)^{3} \psi\left(q^{16}\right) & =-\frac{1}{4} \sum_{n=0}^{\infty}\left(\sum_{d \mid 8 n+5}\left(\frac{2}{d}\right) d\right) q^{8 n+5} \\
q^{7} \psi\left(q^{8}\right) \psi\left(q^{16}\right)^{3} & =\frac{1}{8} \sum_{n=0}^{\infty}\left(\sum_{d \mid 8 n+7}\left(\frac{2}{d}\right) d\right) q^{8 n+7} .
\end{aligned}
$$

PROOF. If $f(q)=\sum_{n=0}^{\infty} c(n) q^{n}$, let us define $[q ; a, b] f(q)$ by

$$
[q ; a, b] f(q)=\sum_{n=0}^{\infty} c(a n+b) q^{a n+b} .
$$

By Lemma 2.1,

$$
\begin{aligned}
& \varphi(-q) \varphi\left(-q^{2}\right) \varphi\left(-q^{4}\right)^{2} \\
& \quad=\left\{\varphi\left(q^{4}\right)-2 q \psi\left(q^{8}\right)\right\}\left\{\varphi\left(q^{8}\right)-2 q^{2} \psi\left(q^{16}\right)\right\}\left\{\varphi\left(q^{8}\right)^{2}-4 q^{4} \psi\left(q^{16}\right)^{2}\right\} .
\end{aligned}
$$


If we expand and extract terms of the form $q^{8 n+1}, q^{8 n+3}, q^{8 n+5}$ and $q^{8 n+7}$, we obtain

$$
\begin{aligned}
{[q ; 8,1] \varphi(-q) \varphi\left(-q^{2}\right) \varphi\left(-q^{4}\right)^{2} } & =-2 q \psi\left(q^{8}\right) \varphi\left(q^{8}\right)^{3} \\
{[q ; 8,3] \varphi(-q) \varphi\left(-q^{2}\right) \varphi\left(-q^{4}\right)^{2} } & =4 q^{3} \psi\left(q^{8}\right) \psi\left(q^{16}\right) \varphi\left(q^{8}\right)^{2} \\
& =4 q^{3} \psi\left(q^{8}\right)^{3} \varphi\left(q^{8}\right) \quad \text { by }(2.8) \\
{[q ; 8,5] \varphi(-q) \varphi\left(-q^{2}\right) \varphi\left(-q^{4}\right)^{2} } & =8 q^{5} \psi\left(q^{8}\right) \varphi\left(q^{8}\right) \psi\left(q^{16}\right)^{2} \\
& =8 q^{5} \psi\left(q^{8}\right)^{3} \psi\left(q^{16}\right) \quad \text { by }(2.8)
\end{aligned}
$$

and

$$
[q ; 8,7] \varphi(-q) \varphi\left(-q^{2}\right) \varphi\left(-q^{4}\right)^{2}=-16 q^{7} \psi\left(q^{8}\right) \psi\left(q^{16}\right)^{3}
$$

respectively. Next, by the first part of Theorem 3.1,

$$
\varphi(-q) \varphi\left(-q^{2}\right) \varphi\left(-q^{4}\right)^{2}=1-2 \sum_{n=1}^{\infty}\left(\sum_{d \mid n}\left(\frac{2}{d}\right) d\right) q^{n}
$$

Therefore, for $1 \leq k \leq 8$,

$$
[q ; 8, k] \varphi(-q) \varphi\left(-q^{2}\right) \varphi\left(-q^{4}\right)^{2}=-2 \sum_{n=0}^{\infty}\left(\sum_{d \mid 8 n+k}\left(\frac{2}{d}\right) d\right) q^{8 n+k}
$$

Using (3.16) in (3.11)-(3.14) we complete the proof.

\section{Combinatorial interpretations}

In this section, we give combinatorial interpretations of the results in Theorems 3.1-3.3. We begin with a lemma.

LEMMA 4.1. Let $n$ be a positive integer and write $n=2^{\alpha} m$, where $m$ is odd and $\alpha$ is a nonnegative integer. Then:

(1) the coefficient of $q^{n}$ in $\varphi(-q) \varphi\left(-q^{2}\right) \varphi\left(-q^{4}\right)^{2}$ is $-2 \sum_{d \mid m}(2 / d) d$;

(2) the coefficient of $q^{n}$ in $q \psi(q)^{2} \psi\left(q^{2}\right) \psi\left(q^{4}\right)$ is $2^{\alpha}(2 / m) \sum_{d \mid m}(2 / d) d$.

PROOF. The first result is immediate from (3.15), together with the fact that $(2 / d)=0$ if $d$ is even. To prove the second result, observe that from Theorem 3.1,

$$
q \psi(q)^{2} \psi\left(q^{2}\right) \psi\left(q^{4}\right)=\sum_{j=1}^{\infty} \frac{j\left(q^{j}-q^{3 j}-q^{5 j}+q^{7 j}\right)}{1-q^{8 j}}=\sum_{j=1}^{\infty} \sum_{k=1}^{\infty}\left(\frac{2}{k}\right) j q^{j k}
$$


The coefficient of $q^{n}$ is therefore

$$
\begin{aligned}
\sum_{j k=n}\left(\frac{2}{k}\right) j & =\sum_{\substack{j k=n \\
k \text { odd }}}\left(\frac{2}{k}\right) j \\
& =\sum_{i k=m}\left(\frac{2}{k}\right) 2^{\alpha} i \\
& =2^{\alpha}\left(\frac{2}{m}\right) \sum_{i k=m}\left(\frac{2}{i}\right) i \\
& =2^{\alpha}\left(\frac{2}{m}\right) \sum_{d \mid m}\left(\frac{2}{d}\right) d,
\end{aligned}
$$

where the multiplicative property of the Jacobi symbol was used in the penultimate step.

Let $a, b, c$ and $d$ be positive integers. Let $r_{(a, b, c, d)}(n)$ denote the number of solutions of $a x_{1}^{2}+b x_{2}^{2}+c x_{3}^{2}+d x_{4}^{2}=n$ in integers (positive, negative and/or zero). The generating function is

$$
\sum_{n=0}^{\infty} r_{(a, b, c, d)}(n) q^{n}=\varphi\left(q^{a}\right) \varphi\left(q^{b}\right) \varphi\left(q^{c}\right) \varphi\left(q^{d}\right)
$$

THEOREM 4.2. If $n$ is a positive integer, write $n=2^{\alpha} m$, where $m$ is odd and $\alpha$ is a nonnegative integer. Then

$$
\begin{aligned}
& r_{(2,1,1,1)}(n)=\left\{2^{\alpha+3}\left(\frac{2}{m}\right)-2\right\} \sum_{d \mid m}\left(\frac{2}{d}\right) d, \\
& r_{(2,2,2,1)}(n)=\left\{2^{\alpha+2}\left(\frac{2}{m}\right)-2\right\} \sum_{d \mid m}\left(\frac{2}{d}\right) d, \\
& r_{(4,2,1,1)}(n)=\left\{2^{\alpha+2}\left(\frac{2}{m}\right)-1-(-1)^{n}\right\} \sum_{d \mid m}\left(\frac{2}{d}\right) d, \\
& r_{(4,4,2,1)}(n)=\left\{2^{\alpha+1}\left(\frac{2}{m}\right)-1-(-1)^{n}\right\} \sum_{d \mid m}\left(\frac{2}{d}\right) d .
\end{aligned}
$$

Proof. Use Lemma 4.1 to extract the coefficient of $q^{n}$ from each of the results in Theorem 3.2.

THEOREM 4.3. Let $o_{j}$ and $e_{j}$ denote an odd integer and an even integer, respectively. For a positive integer $n$, let $A(n), B(n), C(n), D(n)$ and $E(n)$ denote the number of 
solutions of

$$
\begin{aligned}
o_{1}^{2}+2 e_{2}^{2}+2 e_{3}^{2}+2 e_{4}^{2} & =n, \\
o_{1}^{2}+o_{2}^{2}+o_{3}^{2}+2 e_{4}^{2} & =n, \\
o_{1}^{2}+o_{2}^{2}+o_{3}^{2}+2 o_{4}^{2} & =n, \\
o_{1}^{2}+2 o_{2}^{2}+2 o_{3}^{2}+2 o_{4}^{2} & =n
\end{aligned}
$$

and

$$
o_{1}^{2}+o_{2}^{2}+2 o_{3}^{2}+4 o_{4}^{2}=n
$$

respectively. Then

$$
\sum_{d \mid n}\left(\frac{2}{d}\right) d=\left\{\begin{aligned}
\frac{1}{2} A(n) & \text { if } n & \equiv 1(\bmod 8), \\
-\frac{1}{4} B(n) & \text { if } n & \equiv 3(\bmod 8), \\
-\frac{1}{4} C(n) & \text { if } n & \equiv 5(\bmod 8), \\
\frac{1}{2} D(n) & \text { if } n & \equiv 7(\bmod 8) .
\end{aligned}\right.
$$

Since $A(n), B(n), C(n)$ and $D(n)$ are zero unless $n \equiv 1,3,5$ or 7 , respectively, (4.1) completely determines the values of $A(n), B(n), C(n)$ and $D(n)$.

If we write $n=2^{\alpha} m$, where $m$ is odd and $\alpha$ is a nonnegative integer, then

$$
E(n)=2^{\alpha+1}\left(\frac{2}{m}\right) \sum_{d \mid n}\left(\frac{2}{d}\right) d \quad \text { if } n \equiv 0(\bmod 8),
$$

and $E(n)=0$ otherwise.

Proof. Observe that

$$
\begin{aligned}
\sum_{n=0}^{\infty} A(n) q^{n} & =\sum_{x_{1}, x_{2}, x_{3}, x_{4}=-\infty}^{\infty} q^{\left(2 x_{1}+1\right)^{2}+2\left(2 x_{2}\right)^{2}+2\left(2 x_{3}\right)^{2}+2\left(2 x_{4}\right)^{2}} \\
& =2 q \psi\left(q^{8}\right) \varphi\left(q^{8}\right)^{3} .
\end{aligned}
$$

Similarly, we find that

$$
\begin{aligned}
& \sum_{n=0}^{\infty} B(n) q^{n}=8 q^{3} \psi\left(q^{8}\right)^{3} \varphi\left(q^{8}\right), \\
& \sum_{n=0}^{\infty} C(n) q^{n}=16 q^{5} \psi\left(q^{8}\right)^{3} \psi\left(q^{16}\right), \\
& \sum_{n=0}^{\infty} D(n) q^{n}=16 q^{7} \psi\left(q^{8}\right) \psi\left(q^{16}\right)^{3}, \\
& \sum_{n=0}^{\infty} E(n) q^{n}=16 q^{8} \psi\left(q^{8}\right)^{2} \psi\left(q^{16}\right) \psi\left(q^{32}\right) .
\end{aligned}
$$


The identity (4.1) follows from these by comparing coefficients using Theorem 3.3, while (4.2) follows from Lemma 4.1.

The results for $C(n), D(n)$ and $E(n)$ in Theorem 4.3 may be re-expressed in terms of sums of triangular numbers. Let $a, b, c$ and $d$ be positive integers, and let $t_{(a, b, c, d)}(n)$ denote the number of solutions in nonnegative integers of

$$
\frac{a x_{1}\left(x_{1}+1\right)}{2}+\frac{b x_{2}\left(x_{2}+1\right)}{2}+\frac{c x_{3}\left(x_{3}+1\right)}{2}+\frac{d x_{4}\left(x_{4}+1\right)}{2}=n .
$$

The generating function for $t_{(a, b, c, d)}(n)$ is

$$
\sum_{n=0}^{\infty} t_{(a, b, c, d)}(n) q^{n}=\psi\left(q^{a}\right) \psi\left(q^{b}\right) \psi\left(q^{c}\right) \psi\left(q^{d}\right) .
$$

THEOREM 4.4. Let $n$ be a positive integer and write $n=2^{\alpha} m$, where $m$ is odd and $\alpha$ is a nonnegative integer. Then

$$
\begin{aligned}
t_{(2,1,1,1)}(n) & =-\frac{1}{4} \sum_{d \mid 8 n+5}\left(\frac{2}{d}\right) d, \\
t_{(2,2,2,1)}(n) & =\frac{1}{8} \sum_{d \mid 8 n+7}\left(\frac{2}{d}\right) d, \\
t_{(4,2,1,1)}(n-1) & =2^{\alpha}\left(\frac{2}{m}\right) \sum_{d \mid n}\left(\frac{2}{d}\right) d .
\end{aligned}
$$

PROOF. From the generating function for $t_{(2,1,1,1)}(n)$ and (4.5),

$$
\sum_{n=0}^{\infty} t_{(2,1,1,1)}(n) q^{8 n+5}=q^{5} \psi\left(q^{8}\right)^{3} \psi\left(q^{16}\right)=\frac{1}{16} \sum_{n=0}^{\infty} C(n) q^{n}
$$

Equating coefficients and applying Theorem 4.3, we find that

$$
t_{(2,1,1,1)}(n)=\frac{1}{16} C(8 n+5)=-\frac{1}{4} \sum_{d \mid 8 n+5}\left(\frac{2}{d}\right) d .
$$

The other two results may be proved similarly.

Analogues of Lagrange's theorem can be deduced from Theorems 4.2 and 4.3.

THEOREM 4.5. Let $n$ be a nonnegative integer. Then $r_{(2,1,1,1)}(n), r_{(2,2,2,1)}(n)$, $r_{(4,2,1,1)}(n)$ and $r_{(4,4,2,1)}(n)$ are all positive, as are $A(8 n+1), B(8 n+3), C(8 n+5)$, $D(8 n+7)$ and $E(8 n+8)$. 
PROOF. Let $m$ be an odd integer, and let $m=\prod_{p} p^{\alpha_{p}}$ be its prime factorization. By geometric series,

$$
\left(\frac{2}{m}\right) \sum_{d \mid m}\left(\frac{2}{d}\right) d=\prod_{p} \frac{p^{\alpha_{p}+1}-(2 / p)^{\alpha_{p}+1}}{p-(2 / p)},
$$

which is clearly positive. The truth of the theorem follows from this, together with the results in Theorems 4.2 and 4.3. The trivial observation

$$
r_{(2,1,1,1)}(0)=r_{(2,2,2,1)}(0)=r_{(4,2,1,1)}(0)=r_{(4,4,2,1)}(0)=1
$$

takes care of the case $n=0$.

REMARK 4.6. It is interesting to compare the results for $r_{\lambda}(n)$ with the corresponding results for $t_{\lambda}(n)$ in Theorem 4.4. We find that

$$
r_{(2,1,1,1)}(8 n+5)=-10 \sum_{d \mid 8 n+5}\left(\frac{2}{d}\right) d=40 t_{(2,1,1,1)}(n)
$$

and

$$
r_{(2,2,2,1)}(8 n+7)=2 \sum_{d \mid 8 n+7}\left(\frac{2}{d}\right) d=16 t_{(2,2,2,1)}(n) .
$$

These are instances of a theorem in [1], which says that $r_{\left(\lambda_{1}, \ldots, \lambda_{k}\right)}\left(8 n+\lambda_{1}+\cdots+\lambda_{k}\right)$ is a constant (independent of $n$ ) multiple of $t_{\left(\lambda_{1}, \ldots, \lambda_{k}\right)}(n)$, provided $\lambda_{1}+\cdots+\lambda_{k}<8$. Furthermore,

$$
\begin{aligned}
r_{(4,2,1,1)}(8 n)-r_{(4,2,1,1)}(2 n) & =\left(2^{\alpha+5}-2^{\alpha+3}\right)\left(\frac{2}{m}\right) \sum_{d \mid m}\left(\frac{2}{d}\right) d \\
& =24 \times 2^{\alpha}\left(\frac{2}{m}\right) \sum_{d \mid m}\left(\frac{2}{d}\right) d \\
& =24 t_{(4,2,1,1)}(n-1),
\end{aligned}
$$

and this is an instance of a theorem in [2].

\section{References}

[1] C. Adiga, S. Cooper and J. H. Han, 'A general relation between sums of squares and sums of triangular numbers', Int. J. Number Theory 1 (2005), 175-182.

[2] N. D. Baruah, S. Cooper and M. Hirschhorn, 'Sums of squares and sums of triangular numbers induced by partitions of 8', Int. J. Number Theory to appear.

[3] B. C. Berndt, Ramanujan's Notebooks, Part III (Springer-Verlag, New York, 1991).

[4] S. H. Chan, 'Generalized Lambert series identities', Proc. London Math. Soc. (3) 91 (2005), 598622.

[5] J. M. Dobbie, 'A simple proof of some partition formulae of Ramanujan's', Quart. J. Math. Oxford Ser. (2) 6 (1955), 193-196. 
[6] N. J. Fine, Basic Hypergeometric Series and Applications (American Mathematical Society, Providence, RI, 1988).

[7] E. T. Whittaker and G. N. Watson, A Course of Modern Analysis, 4th edn (Cambridge University Press, Cambridge, 1927).

SHAUN COOPER, Institute of Information and Mathematical Sciences, Massey University, Private Bag 102904, North Shore Mail Centre, Auckland, New Zealand

e-mail: s.cooper@massey.ac.nz 